\title{
A Uniform and Reduced Mathematical Model for Sucker Rod Pumping
}

\author{
Leiming Liu ${ }^{1}$, Chaonan Tong ${ }^{1}$, Jianqin Wang ${ }^{2}$, and Ranbing Liu ${ }^{3}$ \\ ${ }^{1}$ University of Science and Technology in Beijing, 100083, China \\ leiming.liu@163.com \\ ${ }^{2}$ Laboratory of Remote Sensing Information Sciences, Institute of Remote Sensing \\ Applications, Chinese Academy of Sciences, P. Box 9718, Beijing 100101, China \\ ${ }^{3}$ University of Petroleum in Beijing, 102249, China
}

\begin{abstract}
The dynamic mathematical models were greatly developed to describe the motion of the sucker-rod pumping system since 1960s and various mathematic models based on different assumptions were presented. This paper does a study in three sides. First, a mathematical model is presented to describe the sucker-rod string with longitudinal and transverse vibrations, and coupled with longitudinal vibration of the tubing and fluid columns in a deviated well. Second, the relations of several kinds of mathematical models in sucker-rod pumping systems are discussed, and the model made in this paper, when based on different assumptions, can become different models made by people these years, which are presented in important references. Third, a method of characteristics is used to transform the set of partial differential equations which describe the vibration of the sucker-rod string, and coupled with vibrations of tubing and liquid columns in the sucker-rod pumping system. Through the transformation, a diagonal partial differential equations set is obtained. Hence a relatively complex model is transformed into a reduced model which is easy to solve. This model has basic meaning for using the technique of pattern recognition to make automatic diagnosis of sucker rod pumping system.
\end{abstract}

\section{Introduction}

Rod pumping is still the most widely used means for artificial lift in oil wells, so ever since 1960s, people lay great emphasis on the mathematical methods of predicting, designing and diagnosis of the sucker-rod pumping systems.

Gibbs(1963)[1] made a 1D mathematical model for the vibration of the sucker rod, which is comprised with a second-order partial differential equation including boundary conditions. Doty and Schmidt(1983)[2] presented a composite model in which both rod string dynamics and fluid dynamics are coupled to account for viscous damping, which was comprised of four first-order equations with four unknown variables, boundary conditions and initial conditions. In the paper of Wang et al. (1992)[3], a set of six equations governing the vibration of the sucker-rod string, tubing and liquid columns in the sucker-rod pumping system are presented.

All these three models stated above are for vertical wells. At present many wells are designed as deviated wells. Lukasiewicz(1991)[4] presented a model of sucker- 
rod string with longitudinal and transverse displacements in a inclined well. Gibbs (1992)[5] studied the situation that sucker rod is crooked, but he assumed the transverse displacement was ignored, for the transverse displacement of the rod was constrained by the tubing. The wave equation about the rod with longitudinal displacement that Gibbs presented took the crooked wells into consideration. $\mathrm{Xu}$ and Mo(1993, 1995)[6,7] made a 3D model of vibrations of sucker-rod string. Li et al. (1999)[8] made a set of equations describing the 3D vibration of the sucker rod beam pumping system.

The models stated above didn't take the movement of the fluid column into consideration. On the basis of the work stated above, this paper presents a set of firstorder partial differential equations and the concerning boundary and initial conditions. The equations describe the $2 \mathrm{D}$ vibration of rod string, the longitudinal vibration of tubing column and coupled with the displacement of the fluid column in the directional and horizontal wells. In these equations, three of them are geometry equations can be solved through the method dealing with non-dynamic problems, the other six first-order partial differential equations are a set of quasi-linear hyperbolic equations, they are dynamic equations, but can be reduced to diagonal equations, that is to make each equation be a partial deviation equation containing only one unknown variable.

\section{Analysis of the Geometry Relations and Forces Acting on the Spatially Curved Rod}

\subsection{Geometry Equations of Spatial Curve}

We build a spatial rectangular coordinates END (see Fig.1 )for the spatial crooked sucker-rod at the well bore, take a rod element of length $\Delta \mathrm{S}$ and build a natural coordinates $\mathrm{t}_{1} \mathrm{t}_{2} \mathrm{t}_{3}$ at the center of $\Delta \mathrm{s}$. $\mathrm{t}_{1}$ is the tangent unit vectors of the element $\Delta \mathrm{s}$, $\mathrm{t}_{2}$ is the principal unit normal vectors and $t_{3}$ is the subnormal unit normal vectors. The Frenet equations is:

$$
\left\{\begin{array}{c}
\boldsymbol{t}_{1}=\mathrm{d} \boldsymbol{r} / \mathrm{d} s \\
\mathrm{~d} \boldsymbol{t}_{1} / \mathrm{d} s=k \boldsymbol{t}_{2} \\
\mathrm{~d} \boldsymbol{t}_{2} / \mathrm{d} s=-k \boldsymbol{t}_{1}+\tau \boldsymbol{t}_{3} \\
\mathrm{~d} \boldsymbol{t}_{3} / \mathrm{d} s=-\tau \boldsymbol{t}_{2}
\end{array}\right.
$$

where $k, \tau$ are the curvature and buckling of the well axis curve and $\boldsymbol{r}$ the position vector of the point on

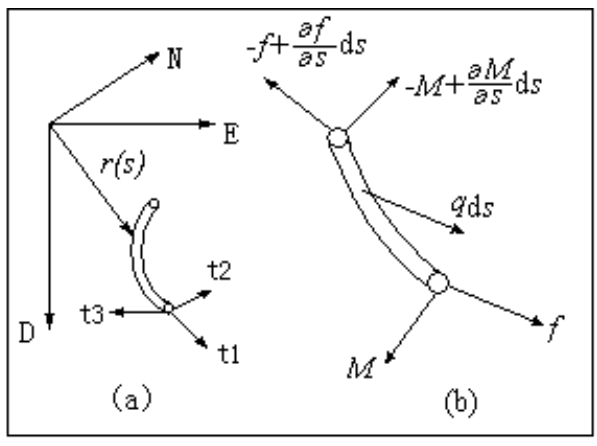

Fig. 1. Forces on rod element the curve. 


\subsection{The Analysis of Forces Acting on the Element of Curved Rod}

Let the internal forces, force moments and external forces acting on element of the curved rod and the displacement of the centroid of the element in the natural coordinates respectively be presented as follows:

$$
\left\{\begin{array}{c}
\boldsymbol{f}=\left(f_{1}, f_{2}, f_{3}\right)^{T}, \boldsymbol{M}=\left(M_{1}, M_{2}, M_{3}\right)^{T} \\
\boldsymbol{q}=\left(q_{1}, q_{2}, q_{3}\right)^{T}, \boldsymbol{u}=\left(u_{1}, u_{2}, u_{3}\right)^{T}
\end{array}\right.
$$

We can obtain the following force equilibrium equations and moment equilibrium equations:

$$
\left\{\begin{array}{c}
\partial \boldsymbol{f} / \partial s+\boldsymbol{q}=m \partial^{2} \boldsymbol{u} / \partial t^{2} \\
\partial \boldsymbol{M} / \partial s=\boldsymbol{f} \times \boldsymbol{t}_{\boldsymbol{I}}
\end{array}\right.
$$

where $m$ is the mass of unit length $\operatorname{rod}(\mathrm{kg})$, and $m=A \rho$, where $A$ is the rod crosssectional area $\left(\mathrm{m}^{2}\right), \rho$ is the rod material density $\left(\mathrm{kg} / \mathrm{m}^{3}\right)$. Let $E_{A}$ be dragging stiffness of the rod $\left(\mathrm{N} \cdot \mathrm{m}^{2}\right), E_{I}$ be bending stiffness of the rod $(\mathrm{N})$. If the effects of twisting moment are ignored, the constitutive equations describing the relations between the internal forces acting on the rod element of length and deformation are obtained:

$$
\left\{\begin{array}{c}
f=E_{A}\left(\frac{\partial u_{1}}{\partial s}-k u_{2}\right) \\
M_{2}=E_{I}\left(-\frac{\partial^{2} u_{3}}{\partial s^{2}}-\tau \frac{\partial u_{2}}{\partial s}\right) \\
M_{3}=E_{I}\left(\frac{\partial^{2} u_{2}}{\partial s^{2}}-\tau \frac{\partial u_{3}}{\partial s}+k\right)
\end{array}\right.
$$

\section{Partial Differential Equations Describing the Movement of Suck-Rod String and Tubing and Fluid Columns in the Directional and Horizontal Wells}

\subsection{Fundamental Assumptions}

(1) The well bore is considered as planar curve.

(2) The sucker-rod only has longitudinal and transverse vibrations in a plane, i.e. $u_{3}=0$.

(3) The cross-section of the sucker rod is round.

(4) The sucker rod string is concentric with the well bore.

According to these assumptions, from Eq. (4), we get $\mathrm{M}_{2}=0$, from the second equation of (3), we get $\frac{\partial M_{1}}{\partial s}=0$. 


\subsection{Partial Differential Equations Describing Motions of Rod String and Tubing Column}

Based on fundamental assumptions, we expand Eq.(3) and take the derivative of the second equation of Eq.(3) with respect to s, then apply the principal of vector derivation and Eq.(4), we obtain:

$$
\left\{\begin{array}{c}
\frac{\partial f_{r 1}}{\partial s}+q_{r 1}=m_{r} \frac{\partial v_{r 1}}{\partial t},\left(\frac{\partial u_{r 1}}{\partial t}=v_{r 1}\right) \\
\frac{\partial f_{r 1}}{\partial t}=E_{A r}\left(\frac{\partial v_{r 1}}{\partial s}-k_{r} v_{r 2}\right) \\
\frac{\partial f_{r 2}}{\partial s}+q_{r 2}=m_{r} \frac{\partial v_{r 2}}{\partial t},\left(\frac{\partial u_{r 2}}{\partial t}=v_{r 2}\right) \\
k f_{r 1}-\frac{\partial f_{r 2}}{\partial s}=E_{I r} \frac{\partial^{2}}{\partial s^{2}}\left(\frac{\partial^{2} u_{2}}{\partial s^{2}}+k\right)
\end{array}\right.
$$

where $k_{r}$ is the plane curvature of the $\operatorname{rod}(1 / \mathrm{m}), m_{r}$ is mass of the unit length of the sucker rod $(\mathrm{kg}), E_{A r}$ the dragging stiffness $(\mathrm{N}), E_{I r}$ the bending stiffness $\left(\mathrm{N} \cdot \mathrm{m}^{2}\right)$.

The curvature causes the lateral displacements of the rods between the two guides, so the relations between the transverse displacement and internal force of the sucker rod can be described by the last two equations of Eq.(5). As for the tubing, it has the same expressions as Eq.(5).

Considering the computing precision of the moment of the tubing, we ignore the transverse displacement of the tubing, the last two equations of Eq.(5) can become a geometry equation. In the following discussion the subscribe $t$ refer to tubing property, then we can obtain the differential equations describing vibrations of the tubing:

$$
\left\{\begin{array}{l}
\frac{\partial f_{t}}{\partial s}+q_{t 1}=m_{t} \frac{\partial v_{t}}{\partial t},\left(\frac{\partial u_{t}}{\partial t}=v_{t}\right) \\
\frac{\partial f_{t}}{\partial t}=E_{A t} \frac{\partial v_{t}}{\partial s} \\
k_{t} E_{A t} \frac{\partial u_{t}}{\partial s}+q_{t 2}=E_{I t} \frac{\mathrm{d}^{2} k_{t}}{\mathrm{~d} s^{2}}
\end{array}\right.
$$

\subsection{D Flow Equation of the Fluid}

As for the flowing fluid of the tubing, we assume fluid column contains no gas, though the well bore is a curve, the movement of the fluid is still 1D flow. Let $\rho_{f}$ be the density of the fluid $\left(\mathrm{kg} / \mathrm{m}^{3}\right)$ at the point of $s$ and at the time of $t$; and the functions of velocity $(\mathrm{m} / \mathrm{s})$ and pressure $\left(\mathrm{N} / \mathrm{m}^{2}\right)$ of the fluid are respectively represented by the variables of $v_{f}$ and $p_{f}$. So the equation of continuity of the fluid, Euler motion equation and the state equation are presented as follows: 


$$
\left\{\begin{array}{l}
\frac{\partial \rho_{f}}{\partial t}+\rho_{f} \frac{\partial v_{f}}{\partial s}+v_{f} \frac{\partial \rho_{f}}{\partial s}=0 \\
\frac{\partial v_{f}}{\partial t}+v_{f} \frac{\partial v_{f}}{\partial s}+\frac{1}{\rho_{f}} \frac{\partial p_{f}}{\partial s}=\frac{q_{f}}{\rho_{f}\left(A_{t}-A_{r}\right)} \\
p_{f}=p_{f}\left(\rho_{f}\right)
\end{array}\right.
$$

where $q_{f}$ is the external forces acting on the fluid column of per unit length (including forces by the sucker rod string, rod coupling and tubing ), $A_{r}$ is the crosssectional area of the sucker rod and $A_{t}$ the inner cross-sectional area of the tubing $\left(\mathrm{m}^{2}\right)$. The equation of fluid state shows that the pressure $p_{f}$ is only related with density $\rho_{f}$, and according to the physical meaning, $\frac{\mathrm{d} p}{\mathrm{~d} \rho}>0$, so define

$$
\frac{\mathrm{d} \rho_{f}}{\mathrm{~d} p_{f}}=a\left(\rho_{f}\right)^{2}
$$

$\rho_{f}$ has the representation:

$$
\rho_{f}=\rho_{0}{ }^{C_{\rho}}\left(p_{f}-p_{0}\right)
$$

or the approximate expression of Eq. (9):

$$
\rho_{f}=\rho_{0}\left[1+C_{\rho}\left(p_{f}-p_{0}\right)\right]
$$

where $\rho_{0}, p_{0}$ are respectively the density and pressure of the fluid in the standard state, $C_{\rho}$ is the relative increment of the density when the pressure increases one unit, i.e. elastic condensation coefficient of the density of the fluid. Let

$$
Q_{f}=q_{f} / \rho_{f}\left(A_{t}-A_{r}\right)
$$

Using Eqs. (8) and (11), Eq. (7) becomes the following equations:

$$
\left\{\begin{array}{c}
\frac{\partial \rho_{f}}{\partial t}+v_{f} \frac{\partial \rho_{f}}{\partial s}+\rho_{f} \frac{\partial v_{f}}{\partial s}=0 \\
\frac{\partial v_{f}}{\partial s}+v_{f} \frac{\partial v_{f}}{\partial s}+\frac{a^{2}\left(\rho_{f}\right)}{\rho_{f}} \frac{\partial \rho_{f}}{\partial s}=Q_{f}
\end{array}\right.
$$

\section{Analysis of External Forces Acting on the Rod String and Tubing and Fluid Columns}

Through the effects of the fluid viscosity resistance, lateral extrusion stress, coulomb friction and gravity caused by the periodic motion of the sucker rod, the sucker rod 
string, tubing column and fluid column comprises a system of motion. These forces are expressed as follows:

$$
\begin{aligned}
& q_{r 1}=-\eta v_{r 1}-\lambda\left|q_{N}\right| \operatorname{sign}\left(v_{r 1}\right)+m g \cos \alpha \\
& q_{r 2}=-\eta v_{r 2}+q_{N} \cos \varphi-m g \sin \alpha
\end{aligned}
$$

where $\eta$ is viscous damping coefficient of the fluid, $m_{r}$ is the mass of the unit length rod, $\varphi$ is the angle between lateral extrusion stress and the principal normal vector, $\lambda$ is friction factor, $q_{N}$ is lateral extrusion stress between the rod and the tubing[7], $\alpha$ is the angle between the tangent of the element of the curved rod and vertical. Here the viscosity resistance is comprised of two parts, one is the viscosity resistance between fluid and the sucker rod, the other between the fluid and the coupling of the rod, and also:

$$
\begin{aligned}
& q_{t 1}=-\eta v_{t}-\lambda\left|q_{N}\right| \operatorname{sign}\left(v_{t}\right)+m g \cos \alpha \\
& q_{t 2}=q_{N} \cos \varphi-m_{t} g \sin \alpha
\end{aligned}
$$

where $\eta, \lambda, q_{N}$ is the same as which is stated above, but the difference is there is no term of viscous resistance in Eq. (16), and $m_{t}$ is the mass of the unit length rod.

The force $q_{f}$ in Eq. (7) has the representation as follows:

$$
q_{f}=-\rho_{f}\left(A_{t}-A_{r}\right) g+\eta v_{r 1}+\eta v_{t}
$$

$\eta v_{r 1}, \eta v_{t}$ of Eq. (17) has the contrary sign with the corresponding term of Eqs. (13) and (15), this shows that the viscous resistance acting on the rod string and tubing column has the reaction to the fluid.

\section{Boundary and Initial Conditions}

The boundary and initial conditions are very important, the former determines if the solution can correctly describe the motion stated, and the correct choice of the latter can make the computing program converges to its periodic numeric solution of stationary state as fast as possible. Papers [1], [2], [3] and [7] made deep study about the boundary and initial conditions and presented useful conclusions. This paper applies those results.

As for the diagnosis, the surface boundary conditions are

$$
\left\{\begin{array}{c}
f_{r 1}(0, t)=f_{1}(t), v_{r 1}(0, t)=v_{1}(t), v_{r 2}(0, t)=0, f_{r 2}(0, t)=0 \\
v_{t}(0, t)=0, f_{t}(0, t)=0, v_{r 2}\left(s_{i}, t\right)=0, p_{f}(0, t)=p_{0}(t)
\end{array}\right.
$$

where $f_{1}(t)$ is the measured loading function of the polished rod, $v_{1}(t)$ is the velocity of the polished $\operatorname{rod}, s_{i}(i=1,2, \cdots, N)$ represents placing a rod guide where $s=s_{i}$, and 
$p_{0}$ represents tubing head pressure and is constant in most of the cases. As for the predicted model, we should consider the boundary conditions in the place of oil-well pump, see paper [3].

The correct choice of the initial conditions can make the computing program converges to its periodic numeric solution of stationary state as fast as possible, so refer to papers [2] and [3], we make discrete transform along the axes of the well for the initial conditions given in the papers, and obtain the initial conditions for this model.

\section{Characteristic Transform of the Partial Differential Equations Set}

The first two equations of Eq. (5), Eq. (6) and Eq.(12) comprise a equations set about the unknown variables of $v_{r 1}, f_{r 1}, v_{t}, f_{t}, v_{f}$ and $\rho_{f}$. We apply the theory of characteristic for the quasi-linear hyperbolic partial differential equations set to transform the six equations into a diagonal equations set about the variables of $w_{1}, w_{2}, w_{3}, w_{4}, w_{5}$ and $w_{6}$.

$$
\left\{\begin{array}{c}
\frac{\partial w_{1}}{\partial t}-\sqrt{\frac{E_{A r}}{\rho_{r}}} \frac{\partial w_{1}}{\partial s}=\frac{q_{r 1}}{\rho_{r} A_{r}}, \frac{\partial w_{2}}{\partial t}+\sqrt{\frac{E_{A r}}{\rho_{r}}} \frac{\partial w_{2}}{\partial s}=\frac{q_{r 1}}{\rho_{r} A_{r}} \\
\frac{\partial w_{3}}{\partial t}-\sqrt{\frac{\rho_{t}}{E_{A t}}} \frac{\partial w_{3}}{\partial s}=\frac{q_{t}}{\rho_{t}\left(A_{h}-A_{t}\right)}, \frac{\partial w_{4}}{\partial t}+\sqrt{\frac{\rho_{t}}{E_{A t}}} \frac{\partial w_{4}}{\partial s}=\frac{q_{t}}{\rho_{t}\left(A_{h}-A_{t}\right)} \\
\frac{\partial w_{5}}{\partial t}+\left(v_{f}+a\right) \frac{\partial w_{5}}{\partial s}=Q_{f}, \frac{\partial w_{6}}{\partial t}+\left(v_{f}-a\right) \frac{\partial w_{6}}{\partial s}=-Q_{f}
\end{array}\right.
$$

where $w_{1}=v_{r 1}+\frac{1}{A_{r} \sqrt{\rho_{r} E_{A r}}} f_{r 1}, w_{2}=v_{r 1}-\frac{1}{A_{r} \sqrt{\rho_{r} E_{A r}}} f_{r 1}, w_{3}=v_{t}+\frac{1}{\sqrt{\rho_{t} E_{A t}}\left(A_{h}-A_{t}\right)} f_{t}$,

$$
w_{4}=v_{t}-\frac{1}{\sqrt{\rho_{t} E_{A t}}\left(A_{h}-A_{t}\right)} f_{t}, w_{5}=v_{f}+\int_{\rho_{f 0}}^{\rho_{f}} \frac{a(z)}{z} \mathrm{~d} z, w_{6}=-v_{f}+\int_{\rho_{f 0}}^{\rho_{f}} \frac{a(z)}{z} \mathrm{~d} z .
$$

where $\rho_{f 0}$ is the density of the fluid when $p=p_{0}$ and $p_{0}$ is the tubing head pressure. The Eq. (19) is easy to solve applying the difference method.

\section{Conclusions}

The model presented in this paper is the basic model for the directional and horizontal wells, it takes the longitudinal and transverse vibrations of the sucker rod into consideration, and also considers the coupled motions of rod string and tubing and fluid columns. So it is a relatively accurate model simulating the dynamic behavior of the sucker-rod system. 
This is a basic model and concentrates the characters of some principal mathematical models of the sucker-rod system. When the curvature of the well bore $k=0$, the mathematical model becomes the model for the coupled vibrations of the rod string and tubing and fluid column in the vertical well[3]. If we further consider the tubing is anchored, and assume the tubing is rigid, the model describing the coupled vibrations of the rod string and fluid column in the vertical well is obtained[2]. If the fluid is assumed as non-condensable, the wave equation of the vibration of the sucker-rod in the vertical well is obtained[1].

As for the mathematical model about the directional and horizontal well, if we assume the fluid is non-condensable, and assume the tubing is anchored and rigid, we can obtain the mathematical model about the $2 \mathrm{D}$ vibration of the sucker rod in the directional and horizontal well[4,5].

Finally, according to the characteristic deformation we have done, as the Eq. (19) shows, it is also easily-solving model.

Acknowledgement. Jianqin Wang thanks Dr. Yong Xue, his PhD supervisor, for his support from the "CAS Hundred Talents Program".

\section{References}

1. Gibbs, S.G., 1963. Predicting the behavior of sucker-rod systems. J. Pet. Technol. (Jul.), pp. 769-778.

2. Doty, D.R. and Schmidt, Z., 1983. An improved model for sucker rod pumping. J. Soc. Pet. Eng. (Feb.), pp. 33-41.

3. Wang, G.W., Rahman,S.S. and Wang, G.Y.,1992.An improved model for sucker rod pumping systems. Proc. 11th. Australas Fluid Mech. Conf., Tasmania, 14-18 Dec., 1992, 2:1137-1140.

4. Lukasiewicz, S.A., 1991.Dynamic behavior of the sucker rod string in the inclined well.SPE 21665,pp.313- 321.

5. Gibbs, S.G., 1992.Design and diagnosis of deviated rod-pumped wells.J. Pet. Technol. (Jul.), pp.774-781.

6. Xu, J., Hu, Y. and U.T.,1993.A method for designing and predicting the sucker rod string in deviated pumping wells.SPE 26929,pp.383-384.

7. Xu, J.and Mo, Y.,1995.Longitudinal and transverse vibration model of the sucker rod string in directional wells and its application in diagnosis.J.Tongji.University.(Feb.), pp.26-30.

8. Li, Z. et al.,1999. Fundamental equations and its applications for dynamical analysis of rod and pipe string in oil and gas wells.ACTA.20(3),pp.87-90. 